\title{
Utilisation des os calcinés dans la défluoruration des eaux contaminées : Détermination expérimentale de la température de calcination et de la granulométrie optimales des os
}

\author{
Léonce Firmin COMLAN DOVONON ${ }^{1,2^{*}}$, Henri Houénoukpo SOCLO ${ }^{2}$, \\ Magloire Acakpo Nonvignon GBAGUIDI ${ }^{2}$ et Alassane YOUSSAO ${ }^{2}$ \\ ${ }^{1}$ Laboratoire d'Analyse des Eaux de la Direction Générale de l'Eau (DG-Eau) 01 BP 385 Cotonou, République \\ du Bénin. \\ ${ }^{2}$ Unité de Recherche en Ecotoxicologie et Etude de Qualité (UREEQ)/Laboratoire de Recherche en Chimie \\ Appliquée (LERCA)/Ecole Polytechnique d'Abomey-Calavi (EPAC)/Université d'Abomey-Calavi (UAC) 01 BP \\ 2009 Cotonou, République du Bénin. \\ *Auteur correspondant, E-mail : leoncedovonon@yahoo.fr/ldovonon@yahoo.fr
}

\section{RESUME}

Le présent travail a consisté à une défluoruration des eaux souterraines de la localité de Essèkpa dans la commune de Dassa au Bénin dont la concentration en ions fluorures est de 6,2 $\mathrm{mg} / \mathrm{L}$. Les essais au laboratoire ont porté sur $250 \mathrm{ml}$ d'eau traitée avec $20 \mathrm{~g}$ d'os de bœuf calcinés à différentes températures $\left(\mathrm{de} 100{ }^{\circ} \mathrm{C}\right.$ à 625 ${ }^{\circ} \mathrm{C}$ ) et à différentes granulométries à savoir os entier, os concassés de granulométries 4 à $6 \mathrm{~mm}$ et 1 à $3 \mathrm{~mm}$. Les aliquotes du milieu réactionnel sont prélevées et dosées toutes les heures jusqu'à 12 heures. Après 12 heures, des observations ont porté sur 24 et 48 heures. Les teneurs des ions fluorures avant et après traitement ont été mesurées par spectrophotométrie d'absorption moléculaire à $580 \mathrm{~nm}$. Les meilleurs résultats expérimentaux ont été obtenus avec les os calcinés à $500{ }^{\circ} \mathrm{C}$ pendant 24 heures de granulométrie comprise entre 1 et $3 \mathrm{~mm}$. Ces essais ont conduit à une concentration résiduelle moyenne de $0,83 \pm 0,065 \mathrm{mg} / \mathrm{L}$ et un taux d'abattement moyen de 86,66 $\pm 1,056 \%$.

(C) 2011 International Formulae Group. All rights reserved.

Mots clés : Défluoruration, os calcinés; eaux souterraines.

\section{INTRODUCTION}

La présence des ions fluorures dans les ressources en eau est d'origine anthropique ou naturelle. Dans les pays moins industrialisés comme les pays africains, l'agriculture est la principale source de contamination anthropique des ressources en eau. L'utilisation agricole intensive d'engrais phosphatés (fluoroapatite), d'insecticides ou d'herbicides contenant des fluorures en tant que constituant essentiel ou sous forme d'impuretés (cryolite, fluorosilicate de baryum) induit généralement une pollution des ressources en eau aux alentours des sols ainsi traités (Mazet, 2002). En dehors des contaminations anthropiques, les contaminations naturelles ont, pour la plupart, une origine géologique (Travi, 1993). C'est le cas des eaux souterraines des réservoirs de granite, de gneiss, de certains bassins sédimentaires ainsi que des eaux géothermales qui concentrent de fortes teneurs en ions 
fluorures. En effet, La fluorine, l'apatite et la fluoroapatite sont les principaux minéraux fluorés des roches sédimentaires (Wedepohl, 1974) tandis que l'apatite, la fluorine, la topaze, les tourmalines, les micas et les amphiboles se retrouvent dans les roches du socle (Koritnig, 1963 ; Boukari, 1982).

Bien que jouant un rôle prophylactique dans l'organisme humain (Chavassieux et Meunier, 1995), la consommation et l'accumulation à long terme des ions fluorures dans l'organisme engendrent des effets néfastes tels que la destruction de l'émail des dents, le développement de maladie telle que l'ostéoporose, de troubles cardiaques, psychiques et même des dégâts génétiques (OMS, 2002 ; Yang et Dluhy, 2002). Ainsi selon cet organisme, la consommation d'une eau dont la concentration en ions fluorures est supérieure à $1,5 \mathrm{mg} / \mathrm{L}$ peut conduire aux effets indésirables ci-dessus cités. Face à de tels risques, il est opportun, si l'on ne dispose pas d'une autre source d'alimentation en eau potable, de pratiquer alors une technique spécifique d'élimination du fluorure. Il existe plusieurs méthodes physico-chimiques classiques d'élimination des fluorures de l'eau: la précipitation avec la chaux (Sorg, 1978 ; Degrémont, 1978 ; Travi, 1993), l'adsorption sur alumine activée ou charbon actif (Xu-Guo-Xun, 1992), l'échange d'ions sur les résines, l'osmose inverse et la nanofiltration (Amor et al., 2001 ; Pontié et al., 2006). Ces techniques classiques de défluoruration paraissent trop onéreuses et difficiles à mettre en œuvre dans les pays moins industrialisés qui disposent de peu de moyens financiers et de cadres qualifiés (Amor et al., 2001). L'élimination des ions fluorures des eaux de consommation par adsorption est actuellement en vogue et porte sur de nombreux matériaux (Pontié et al., 2006) tel que le charbon d'os. Ainsi, le choix d'une technique basée sur la valorisation d'un matériau local (les os de bovin) devient opportun en ce sens qu'il contribue également à assainir le cadre de vie.

Cette technique empirique et embryonnaire a besoin d'être calée sur des bases scientifiques. C'est ainsi que nous avons opté, dans cette étude, de déterminer expérimentalement la température de calcification et la granulométrie optimales des os à utiliser pour les essais de traitement efficace.

\section{MATERIEL ET METHODES \\ Milieu d'étude et site d'échantillonnage}

La Figure 1 présente la carte du milieu d'étude. La présente étude a porté sur les eaux d'un forage de la commune de Dassa-Zoumè dans le département des Collines dont les eaux sont contaminées en ions fluorures (Dovonon, 2004 ; Ahokpossi, 2005). Ce département est compris entre $7^{\circ}$ et $8^{\circ}$ de Latitude Nord et les méridiens $1^{\circ} 60^{\prime}$ et $2^{\circ} 80^{\prime}$ de Longitude Est. Il est situé dans la partie centrale de la République du Bénin, pays de l'Afrique de l'Ouest, dans la zone tropicale entre les parallèles $6^{\circ} 30^{\prime}$ et $12^{\circ} 30^{\prime}$ de Latitude Nord et les méridiens $1^{\circ}$ et $3^{\circ} 40^{\prime}$ de Longitude Est (Boukari, 1982).

\section{Matériel}

Tous les essais ont été effectués dans le laboratoire de la Direction Générale de l'Eau à l'aide des équipements suivants :

Un four carbolite de type carbolite "Aston lane, hope" (Shelffied, S30 2RR) couvrant une gamme de températures allant de 0 à $2000{ }^{\circ} \mathrm{C}$ pour la calcination des os; un spectrophotomètre de type $\mathrm{HACH}, \mathrm{DR} / 2400$ pour les mesures colorimétriques; un multi paramètre de type combo by HANNA (combo pH \& EC) pour mesurer les paramètres physiques tels que la température, le $\mathrm{pH}$, la conductivité, le TDS ; une balance de type OHAUS Explorer Pro Précision 0,1 g de portée $12000 \mathrm{~g}$ pour la pesée des os à calciner; une balance de type "Sartorius" (charge maximale $220 \mathrm{~g}$ ) précision 0,01 et 0,1 mg pour le pesé des os à utiliser pour les essais de traitement; un agitateur magnétique; quatre (04) tamis d'ouverture 1 $\mathrm{mm}, 3 \mathrm{~mm}, 4 \mathrm{~mm}$ et $6 \mathrm{~mm}$; des os de bœuf à calciner; des échantillons d'eau naturelle contaminés à $6,2 \mathrm{mg} / \mathrm{L}$ en ion fluorure prélevés dans la commune de Dassa-Zoumé au niveau du seul forage d'Essèkpa. 


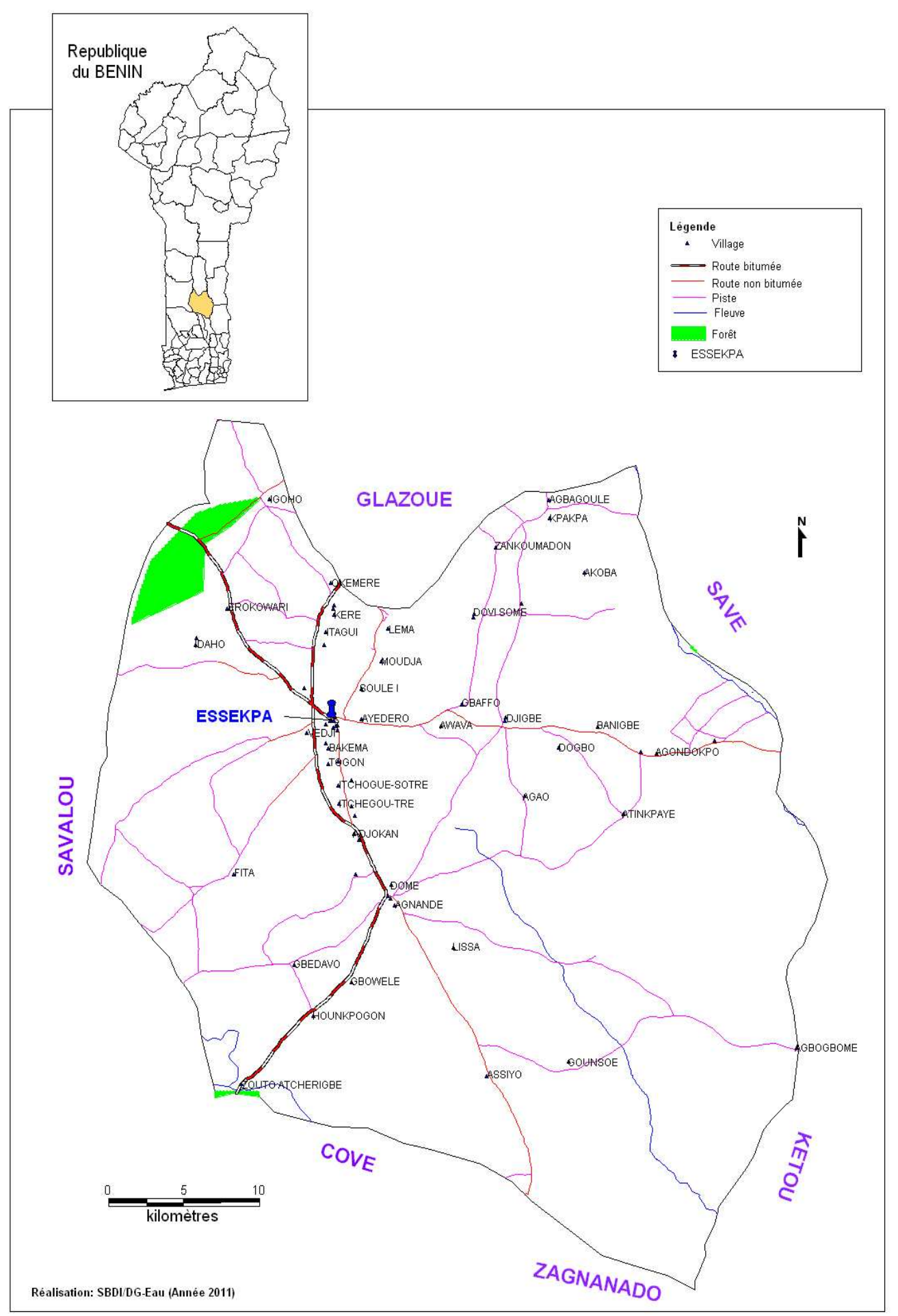

Figure 1 : Localisation de la zone d'étude. 


\section{Calcination des os}

Pour évaluer l'influence de la teneur en eau et de la matière organique de l'os sur l'efficacité du traitement, $24 \mathrm{~kg}$ d'os frais de bœuf récupérés à l'abattoir de Cotonou ont été répartis en 24 échantillons de $1 \mathrm{~kg}$. Vingt et un (21) des vingt quatre échantillons ont été répartis en trois (03) lots de sept (07) échantillons. Chacun des sept (07) échantillons de chaque lot est porté à une température comprise entre $100{ }^{\circ} \mathrm{C}$ et $400{ }^{\circ} \mathrm{C}$ avec un incrément de $50{ }^{\circ} \mathrm{C}$ entre deux températures consécutives. La durée de calcination des trois (03) lots est respectivement de 24 heures $\left(1^{\text {er }}\right.$ lot $), 48$ heures $\left(2^{\text {ème }}\right.$ lot$)$ et 72 heures ( $3^{\text {ème }}$ lot). Deux des trois échantillons restants sont portés à $500{ }^{\circ} \mathrm{C}$ respectivement pendant 24 heures et 48 heures et enfin le dernier échantillon est porté à $625^{\circ} \mathrm{C}$ pendant 24 heures.

Chaque échantillon d'os traité thermiquement a été divisé en quatre parties de $0,125 \mathrm{~kg}$ à savoir : os entier, os concassé et tamisé de granulométrie comprise entre $4 \mathrm{~mm}$ et $6 \mathrm{~mm}$ et enfin $1 \mathrm{~mm}$ et $3 \mathrm{~mm}$.

\section{Essais de défluoruration}

Les travaux ont concerné la préparation et les essais de défluoruration des échantillons d'eau naturelle contaminés à $6,2 \mathrm{mg} / \mathrm{L}$ en ion fluorure prélevés dans la commune de DassaZoumé au niveau du seul forage d'Essèkpa.

Les essais de défluoruration ont été effectués en deux étapes avec $20 \mathrm{~g}$ d'os et 250 $\mathrm{mL}$ d'échantillon d'eau à traiter. Avant les essais de défluoruration, les os traités thermiquement séjournent pendant dix (10) minutes dans de l'eau milli $Q$ afin de minimiser le relargage des éventuels fluorures contenus dans l'os au cours du traitement. Après le lavage, les os sont séchés à $100{ }^{\circ} \mathrm{C}$ pendant également dix (10) minutes. Tous les essais de défluoruration sont répétés trois fois.

\section{Première étape}

Elle a consisté à l'utilisation pour chaque essai des os entier ayant subi l'un des traitements thermiques décrits dans la méthodologie. Ceci à permis d'identifier la température optimale de calcination des os à utiliser pour la suite.

\section{Deuxième étape}

Une fois la température optimale de calcination choisie, la suite des essais de traitement de l'eau contaminée a été effectuée avec des os entiers, des os concassés et tamisés à une granulométrie comprise entre 6 et $4 \mathrm{~mm}$, et, 3 et $1 \mathrm{~mm}$, préalablement portés à cette température.

Les concentrations en ions fluorures avant et après traitement ont été déterminées par colorimétrie d'adsorption moléculaire grâce à l'action du réactif de SPADNS (solution de laque rouge de zirconium de couleur rouge). Il forme avec les ions fluorures un complexe $\mathrm{ZrF}$ incolore. $\mathrm{La}$ coloration résiduelle du réactif de SPADNS est mesurée par spectrophotométrie d'absorption moléculaire avec la DR/2400 à $580 \mathrm{~nm}$ après calibrage. Pour le suivi de la réaction de défluoruration, l'intervalle de temps d'observation est d'une heure pour les douze premières heures. Une observation est faite après 24 heures et une dernière après 48 heures

\section{Analyse statistique}

Les données ont été saisies en Excel et analysées à l'aide du logiciel XLSTAT. Les moyennes (moy) et les écarts type $(\sigma)$ des données enregistrées ont été calculés dans le souci de déterminer les coefficients de variation. Le coefficient de variation $(\mathrm{CV}$ en $\%)$ obtenu à partir de l'expression $\mathrm{CV}=100$ o/moy permet de montrer le degré d'homogénéité des données obtenues.

Ainsi, on montre que (Zogo et al., 2010) :

$\mathrm{Si} \mathrm{CV} \mathrm{<} \mathrm{2 \% ,} \mathrm{les} \mathrm{mesures} \mathrm{sont} \mathrm{très}$ homogènes; Si $2 \%<\mathrm{CV}<30 \%$, les mesures sont homogènes; et

Si CV > 30\%, les mesures sont hétérogènes.

Le test de corrélation de Pearson a été réalisé. Le seuil de signification $\alpha$ de nos résultats est de 0,05 . 


\section{RESULTATS}

Détermination de la température de calcination de l'os

Les Tableaux 1a, $1 \mathrm{~b}$ et $1 \mathrm{c}$ présentent les concentrations résiduelles et les taux d'abattement en ions fluorures obtenus lors des différents essais de défluoruration des eaux contaminées avec $20 \mathrm{~g}$ d'os traités thermiquement à différentes températures. Lorsque la température de calcination varie de $100{ }^{\circ} \mathrm{C}$ à $625^{\circ} \mathrm{C}$ pendant 24 heures (Tableau 1a) les concentrations résiduelles en ion fluorure varient de 0,78 à $2,2 \mathrm{mg} / \mathrm{L}$ après 24 heures de contact de l'eau contaminée avec le matériel osseux utilisé. Après 48 heures de défluoruration les concentrations en ion fluorure de l'eau traitée varient entre 0,39 et $1,5 \mathrm{mg} / \mathrm{L}$. Les taux d'abattement pour ces deux périodes de défluoruration varient respectivement de 64,52 à $87,42 \%$ et de 75,80 à 93,71\%. De 100 à $400{ }^{\circ} \mathrm{C}$, les eaux traitées présentent une coloration jaune, une couche lipidique surnageante et des odeurs. Cet état est illustré par la photo1. A partir de $500{ }^{\circ} \mathrm{C}$ les eaux traitées ne présentent plus de coloration ni d'odeur (Photo 2).

Afin de déterminer l'influence du temps sur la capacité des os calcinés à ne pas générer des couleurs ni d'odeur dans les eaux traitées, les os ont été calcinés entre 100 et $400^{\circ} \mathrm{C}$ respectivement à 48 et 72 heures (Tableaux $1 \mathrm{~b}$ et 1c). Les essais de traitements n’ont pas donnés des résultats satisfaisants.

Les coefficients de variations ( $\mathrm{CV}$ en $\%$ ) obtenus des données des Tableaux 1a, 1b, 1c sont comprises entre 2 et $30 \%$. Ce résultat permet de confirmer l'homogénéité des données obtenues. Par contre, les tests de corrélation de Pearson réalisés sur les Tableaux 1a, 1b, 1c montrent que seuls les résultats obtenus après 24 heures de traitement au niveau du Tableau 1a présentaient une corrélation significative au seuil de 0,05 avec un p-value bilatérale de 0,025 entre la température de calcination et la concentration résiduelle.

Ainsi les graphes des Figures 3 et 4 ont été tracés pour illustrer la variation de la concentration en fluorures en fonction $\mathrm{du}$ temps au cours des expériences menées. Ce choix restrictif est justifié par le fait que les allures des courbes obtenues sont similaires de
100 à $625^{\circ} \mathrm{C}$. Les courbes obtenues ont une allure décroissante avec un palier à partir de 24 heures.

\section{Evolution du pH des eaux traitées au cours du temps}

Les Figures 5 et 6 montrent la variation de la concentration de fluorures et du $\mathrm{pH}$ en fonction du temps de traitement de l'eau riche en fluorures avec 1'os calciné respectivement à $500{ }^{\circ} \mathrm{C}$ et $625^{\circ} \mathrm{C}$.

L'analyse du graphe de la Figure 5 nous permet de distinguer trois périodes dans l'évolution du $\mathrm{pH}$ et de la concentration en fluorures :

Durant les 6 premières heures, les deux paramètres évoluent en sens inverse. Le $\mathrm{pH}$ croît progressivement de 7,81 pour atteindre une valeur maximale de 8,57 pendant que la concentration en ions fluorures diminue de 6,2 à $1,87 \mathrm{mg} / \mathrm{L}$;

Entre 6 heures et 28 heures, les deux paramètres évoluent dans le même sens. Le pH décroît de 8,57 à 8,27 entre 6 heures et 10 heures. De cet instant, il reste constant jusqu'à 24 heures avant de croître à nouveau entre 24 heures et 28 heures. Parallèlement, la concentration en fluorures continue de diminuer pour atteindre la valeur minimale de $0,65 \mathrm{mg} / \mathrm{L}$ après 10 heures de traitement. Cette teneur résiduelle reste constante entre 10 heures et 24 heures avant de remonter jusqu'à $2 \mathrm{mg} / \mathrm{L}$ après 28 heures de traitement.

De 28 heures à 48 heures, les deux paramètres évoluent à nouveau en sens inverse. Le $\mathrm{pH}$ continue de croître pour atteindre la valeur maximale de 8,57 précédemment enregistrée. La concentration en ions fluorures passe de $2 \mathrm{mg} / \mathrm{L}$ à 0,24 $\mathrm{mg} / \mathrm{L}$.

L'analyse du graphe de la Figure 6 montre une évolution temporelle en sens inverse des courbes de $\mathrm{pH}$ et de la concentration résiduelle en ions fluorures de l'eau traitée. Le $\mathrm{pH}$ croît graduellement de 7,81 pour atteindre la valeur de 11,3 après 48 heures de traitement, alors que la concentration en ions fluorures décroît de 6,2 $\mathrm{mg} / \mathrm{L}$ à $0,39 \mathrm{mg} / \mathrm{L}$. De tout ce qui précède, la suite des expériences pour le choix de la granulométrie a été conduite avec des os calcinés à $500{ }^{\circ} \mathrm{C}$ (Tableau 2). 
Détermination de la granulométrie optimale des os calcinés

Le Tableau 2 présente les résultats obtenus lors du traitement réalisé avec différentes granulométries d'os calcinés à savoir : os calcinés entiers, os calcinés divisés de granulométrie $4 \mathrm{~mm} \leq \varnothing \leq 6 \mathrm{~mm}$ et $1 \mathrm{~mm}$ $\leq \varnothing \leq 3 \mathrm{~mm}$, avec $\varnothing$ le diamètre des grains. Les concentrations résiduelles varient respectivement entre 1,5 et $1,25 \mathrm{mg} / \mathrm{L}, 1,19$ $\mathrm{mg} / \mathrm{L}$ et $1,52 \mathrm{mg} / \mathrm{L}$ puis entre $0,75 \mathrm{mg} / \mathrm{L}$ et $0,91 \mathrm{mg} / \mathrm{L}$. Toutes les valeurs de concentrations résiduelles obtenues sont inférieures ou égales aux recommandations de l'OMS (1,5 mg/L). Les moyennes calculées donnent respectivement 1,$38 ; 1,32$ et 0,83 $\mathrm{mg} / \mathrm{L}$ d'ions fluorures restants après 24 heures avec successivement les os calcinés entiers et les os calcinés divisés de diamètres compris entre 4 et $6 \mathrm{~mm}$ puis entre 1 et $3 \mathrm{~mm}$. Le test de corrélation de Pearson des données du Tableau 2 montre que tous les trois traitements présentent des données qui ont une corrélation significative à un seuil de 0,05 et des p-value bilatérales respectives de 0,004; 0,037 et 0,019 . Ainsi tous les trois traitements donnent des résultats concluants c'est-à-dire inférieurs au seuil admissible par l'OMS qui varie de 0,7 à $1,5 \mathrm{mg} / \mathrm{L}$ pour l'eau de boisson suivant la température (OMS, 2004).

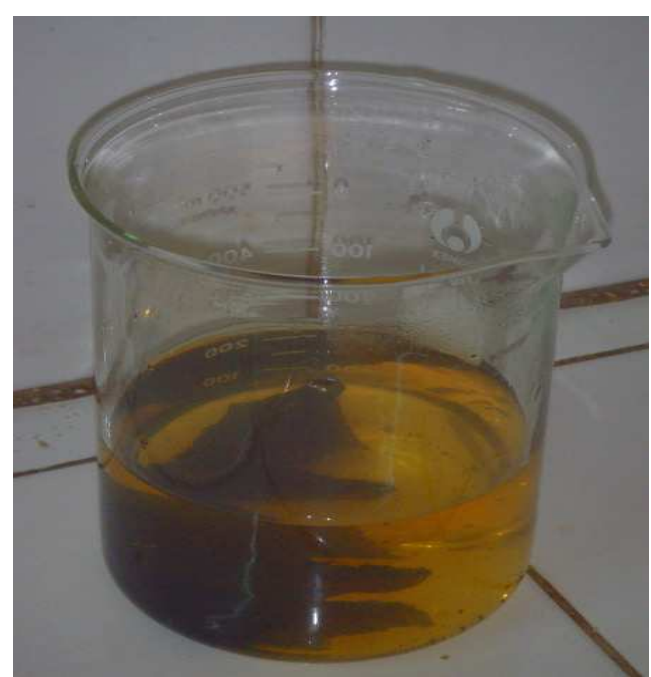

Photo 1 : Etat coloré de l'eau traitée avec des os portés à des températures comprises entre 100 et $400{ }^{\circ} \mathrm{C}$

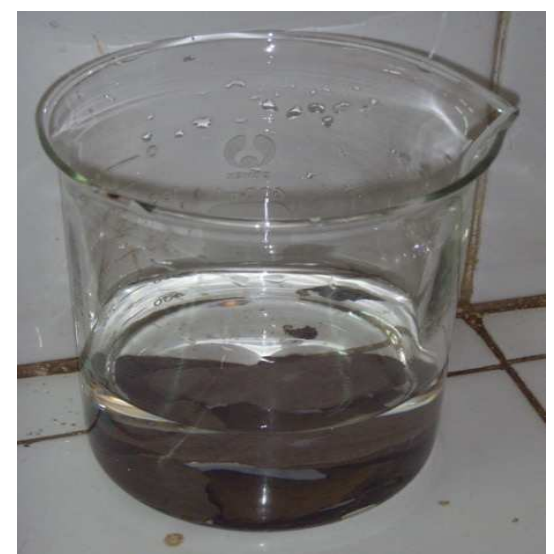

Photo 2 : Etat limpide des eaux traitées avec des os calcinés à des températures entre 100 et supérieures à $400^{\circ} \mathrm{C}$ 




Figure 3 : Variation de la concentration de fluorure en fonction du temps lors du traitement de l'eau fluorée avec $20 \mathrm{~g}$ d'os calciné à $150{ }^{\circ} \mathrm{C}$ pendant 24 heures.

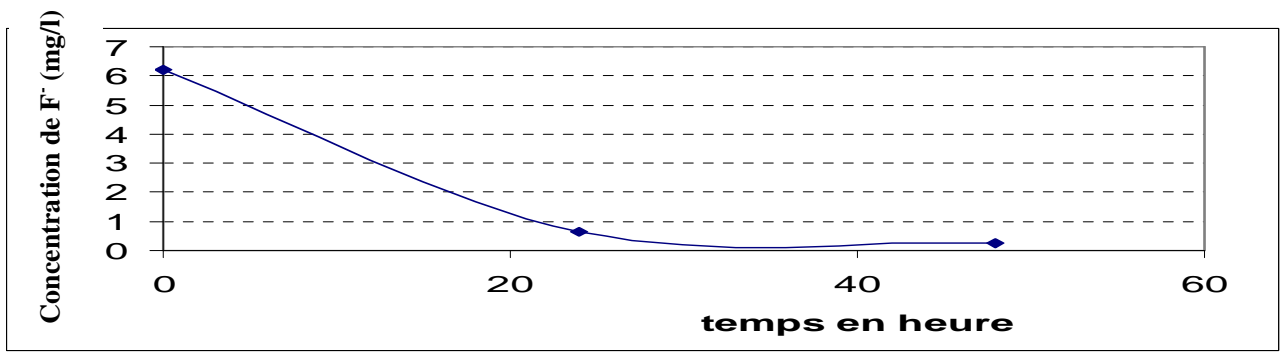

Figure 4 : Variation de la concentration de fluorure en fonction du temps lors du traitement de l'eau fluorée avec $20 \mathrm{~g}$ d'os calciné à $500{ }^{\circ} \mathrm{C}$ pendant 24 heures

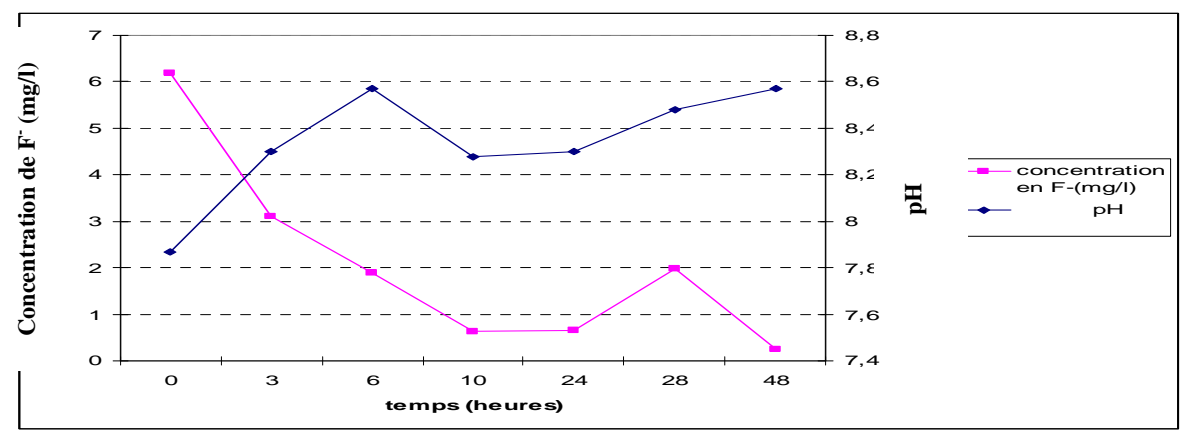

Figure 5 : Variation de la concentration de fluorure et du $\mathrm{pH}$ en fonction du temps lors du traitement de l'eau fluorée avec $20 \mathrm{~g}$ d'os calciné à $500{ }^{\circ} \mathrm{C}$ pendant 24 heures

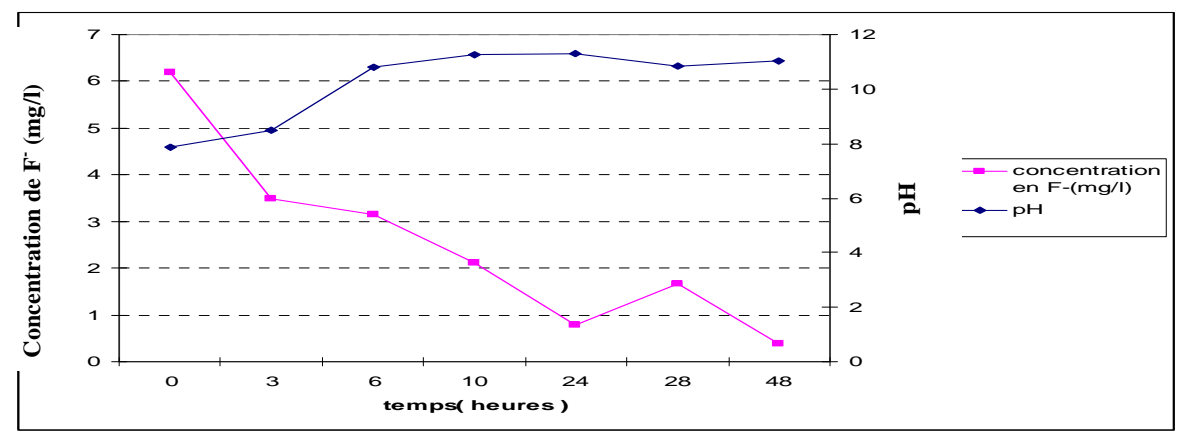

Figure 6 : Variation de la concentration de fluorure et du $\mathrm{pH}$ en fonction du temps lors du traitement de l'eau fluorée avec $20 \mathrm{~g}$ d'os calciné à $600{ }^{\circ} \mathrm{C}$ pendant 12 heures. 


\section{F. COMLAN DOVONON et al. / Int. J. Biol. Chem. Sci. 5(4): 1712-1726, 2011}

Tableau 1a : Résultats des essais de défluoruration de $250 \mathrm{~mL}$ d'eau souterraine contaminée à $6,2 \mathrm{mg} / \mathrm{L}$ avec $20 \mathrm{~g}$ d'os entier calciné pendant 24 heures à différentes températures entre 100 et $625^{\circ} \mathrm{C}$.

\begin{tabular}{|c|c|c|c|c|c|c|c|c|}
\hline $\mathbf{N}^{\circ}$ & $\begin{array}{l}\mathbf{T}^{\circ} \text { de } \\
\text { calcin. } \\
\left({ }^{\circ} \mathbf{C}\right)\end{array}$ & $\begin{array}{l}\text { Moyenne Conc. } \\
\text { en fluorure après } \\
24 \text { heures } \\
(\mathrm{mg} / \mathrm{l})\end{array}$ & $\begin{array}{l}\text { Moyenne Conc. } \\
\text { en fluorures } \\
\text { après 48heures } \\
\text { (mg/L) }\end{array}$ & $\begin{array}{l}\text { Taux } \\
\text { d'abattement } \\
(\%) \text { en } 24 \\
\text { heures } \\
\text { d'exposition }\end{array}$ & $\begin{array}{l}\text { Taux } \\
\text { d'abattement } \\
(\%) \text { en } 48 \\
\text { heures } \\
\text { d'exposition }\end{array}$ & $\begin{array}{l}\text { Coefficient de } \\
\text { variation }(\%) \\
\text { après } 24 \text { heures }\end{array}$ & $\begin{array}{l}\text { Coefficient de } \\
\text { variation }(\%) \\
\text { après } 48 \text { heures }\end{array}$ & Observations \\
\hline 1 & 100 & $1,44 \pm 0,13$ & $1,16 \pm 0,10$ & 76,77 & 81,29 & 9,03 & 8,62 & $\begin{array}{l}\text { Coloration de l'eau } \\
\text { présence de matière } \\
\text { lipidique et } \\
\text { perception d'odeur }\end{array}$ \\
\hline 2 & 150 & $1,93 \pm 0,10$ & $0.43 \pm 0,05$ & 68,87 & 93,06 & 5,18 & 11,63 & Idem \\
\hline 3 & 200 & $1,42 \pm 0,13$ & $1,49 \pm 0,17$ & 77,10 & 75,97 & 9,15 & 11,41 & Idem \\
\hline 4 & 250 & $1,65 \pm 0,10$ & $1,25 \pm 0,16$ & 73,39 & 79,83 & 6,06 & 12,80 & Idem \\
\hline 5 & 300 & $2,2 \pm 0,15$ & $1,26 \pm 017$ & 64,52 & 79,68 & 6,82 & 13,49 & Idem \\
\hline 6 & 350 & $1,75 \pm 0,16$ & $1,50 \pm 0,11$ & 71,77 & 75,80 & 9,14 & 7,33 & Idem \\
\hline 7 & 400 & $1,26 \pm 0,13$ & $0,97 \pm 0,08$ & 79,68 & 84,35 & 10,32 & 8,25 & Idem \\
\hline 8 & 500 & $1,04 \pm 0,16$ & $0.64 \pm 0,05$ & 83,23 & 89,68 & 15,38 & 7,81 & $\begin{array}{l}\text { Pas de coloration ni } \\
\text { de matière lipidique }\end{array}$ \\
\hline 9 & 625 & $0,78 \pm 0,04$ & $0,39 \pm 0,04$ & 87,42 & 93,71 & 5,13 & 10,26 & Idem \\
\hline
\end{tabular}


- Test paramétrique pour les concentrations moyennes en F- après 24 heures

Test de corrélation de Pearson entre les températures de calcination et les concentrations résiduelles enregistrées :

Valeur observée

p-value bilatérale

Alpha

0,05

Conclusion : la corrélation est significative au seuil de 0,05

- Test paramétrique pour les concentrations moyennes en F- après 48 heures

\begin{tabular}{lr}
\hline Test de corrélation de Pearson: & \\
\hline Valeur observée & $-0,438$ \\
p-value bilatérale & 0,277 \\
Alpha & 0,05 \\
\hline \multicolumn{2}{c}{ Conclusion : la corrélation n'est pas significative au seuil de 0,05}
\end{tabular}

- Test paramétrique pour les taux d'abattement après 24 heures

\begin{tabular}{lr}
\hline Test de corrélation de Pearson : \\
\hline Valeur observée & 0,771 \\
p-value bilatérale & 0,025 \\
Alpha & 0,05 \\
\hline Conclusion : la corrélation est significative au seuil de 0,05
\end{tabular}

Conclusion : la corrélation est significative au seuil de 0,05

- $\quad$ Test paramétrique pour les taux d'abattement après 48 heures

\begin{tabular}{lr}
\hline Test de corrélation de Pearson: & \\
\hline Valeur observée & 0,439 \\
p-value bilatérale & 0,276 \\
Alpha & 0,05 \\
\hline \multicolumn{2}{c}{ Conclusion : la corrélation n'est pas significative au seuil de 0,05}
\end{tabular}




\section{F. COMLAN DOVONON et al. / Int. J. Biol. Chem. Sci. 5(4): 1712-1726, 2011}

Tableau 1b : Résultats des essais de défluoruration de $250 \mathrm{ml}$ d'eau souterraine contaminée à $6,2 \mathrm{mg} / \mathrm{L}$ avec $20 \mathrm{~g}$ d'os entiers calcinés pendant 48 heures à différentes températures comprises entre 100 et $400{ }^{\circ} \mathrm{C}$.

\begin{tabular}{|c|c|c|c|c|c|c|c|c|}
\hline $\mathbf{N}^{\circ}$ & $\begin{array}{l}\mathrm{T}^{\circ} \text { de } \\
\text { calcin. } \\
\left({ }^{\circ} \mathrm{C}\right)\end{array}$ & $\begin{array}{l}\text { Moyenne } \\
\text { Conc. en } \\
\text { fluorure après } \\
24 \text { heures } \\
(\mathrm{mg} / \mathrm{l})\end{array}$ & $\begin{array}{l}\text { Moyenne Conc. } \\
\text { en fluorures } \\
\text { après } 48 \text { heures } \\
(\mathrm{mg} / \mathrm{L})\end{array}$ & $\begin{array}{l}\text { Taux } \\
\text { d'abattemen } \\
\text { t }(\%) \text { en } 24 \\
\text { heures } \\
\text { d'exposition }\end{array}$ & $\begin{array}{l}\text { Taux } \\
\text { d'abattement } \\
(\%) \text { en } 48 \\
\text { heures } \\
\text { d'exposition }\end{array}$ & $\begin{array}{l}\text { Coefficient de } \\
\text { variation }(\%) \\
\text { après } 24 \text { heures }\end{array}$ & $\begin{array}{l}\text { Coefficient de } \\
\text { variation }(\%) \text { après } 48 \\
\text { heures }\end{array}$ & Observations \\
\hline 1 & 100 & $1,50 \pm 0,16$ & $1,35 \pm 0,16$ & 75,81 & 78,22 & 10,67 & 11,85 & $\begin{array}{l}\text { Coloration de l'eau } \\
\text { présence de matière } \\
\text { lipidique et perception } \\
\text { d'odeur }\end{array}$ \\
\hline 2 & 150 & $1,37 \pm 0,12$ & $0,54 \pm 0,09$ & 77,90 & 91,3 & 8,76 & 16,67 & Idem \\
\hline 3 & 200 & $1,18 \pm 0,14$ & $1,03 \pm 0,19$ & 80,97 & 83,39 & 11,86 & 18,45 & Idem \\
\hline 4 & 250 & $1,28 \pm 0,13$ & $1,18 \pm 0,12$ & 79,35 & 80,97 & 10,16 & 10,17 & Idem \\
\hline 5 & 300 & $1,21 \pm 0,14$ & $1,8 \pm 0,18$ & 80,48 & 70,97 & 11,57 & 10,00 & Idem \\
\hline 6 & 350 & $1,87 \pm 0,16$ & $1,45 \pm 0,14$ & 69,84 & 76,61 & 8,56 & 9,66 & Idem \\
\hline 7 & 400 & $1,75 \pm 0,16$ & $0,78 \pm 0,06$ & 71,77 & 87,42 & 9,14 & 7,69 & Idem \\
\hline
\end{tabular}

\begin{tabular}{lr}
\hline Test de corrélation de Pearson (test paramétrique) : \\
\hline Valeur observée & 0,709 \\
p-value bilatérale & 0,115 \\
Alpha & 0,05 \\
\hline
\end{tabular}




\section{F. COMLAN DOVONON et al. / Int. J. Biol. Chem. Sci. 5(4): 1712-1726, 2011}

Tableau 1C : Résultats des essais de défluoruration de $250 \mathrm{ml}$ d'eau souterraine contaminée à $6,2 \mathrm{mg} / \mathrm{L}$ avec $20 \mathrm{~g}$ d'os entiers calcinés pendant 72 heures à différente température comprise entre 100 et $400{ }^{\circ} \mathrm{C}$.

\begin{tabular}{|c|c|c|c|c|c|c|c|c|}
\hline $\mathbf{N}^{\circ}$ & $\begin{array}{l}\mathbf{T}^{\circ} \text { de } \\
\text { calcin. } \\
\left({ }^{\circ} \mathbf{C}\right)\end{array}$ & $\begin{array}{l}\text { Moyenne Conc. } \\
\text { en fluorure } \\
\text { après } 24 \text { heures } \\
\text { (mg/l) }\end{array}$ & $\begin{array}{l}\text { Moyenne Conc. } \\
\text { en fluorures } \\
\text { après 48heures } \\
\text { (mg/L) }\end{array}$ & $\begin{array}{l}\text { Taux } \\
\text { d'abattement } \\
(\%) \text { en } 24 \\
\text { heures } \\
\text { d'exposition }\end{array}$ & $\begin{array}{l}\text { Taux } \\
\text { d'abattement } \\
(\%) \text { en } 48 \\
\text { heures } \\
\text { d'exposition }\end{array}$ & $\begin{array}{l}\text { Coefficient de } \\
\text { variation }(\%) \\
\text { après } 24 \text { heures }\end{array}$ & $\begin{array}{l}\text { Coefficient de } \\
\text { variation }(\%) \text { après } \\
48 \text { heures }\end{array}$ & Observations \\
\hline 1 & 100 & $1,23 \pm 0,15$ & $1,10 \pm 0,10$ & 80,16 & 82,25 & 12,20 & 9,09 & $\begin{array}{l}\text { Coloration de l'eau } \\
\text { présence de matière } \\
\text { lipidique } \\
\text { perception d'odeur }\end{array}$ \\
\hline 2 & 150 & $1,21 \pm 0,12$ & $0,9 \pm 0,05$ & 80,48 & 83,39 & 9,92 & 5,56 & Idem \\
\hline 3 & 200 & $1,85 \pm 0,18$ & $1,43 \pm 0,13$ & 70,16 & 76,93 & 9,73 & 9,09 & Idem \\
\hline 4 & 250 & $1,15 \pm 0,15$ & $1,10 \pm 0,19$ & 81,45 & 82,26 & 13,04 & 17,27 & Idem \\
\hline 5 & 300 & $1,85 \pm 0,12$ & $0.20 \pm 0,02$ & 70,16 & 96,77 & 6,49 & 10,00 & Idem \\
\hline 6 & 350 & $2,10 \pm 0,12$ & $0,85 \pm 0,05$ & 66,13 & 86,29 & 5,71 & 5,88 & Idem \\
\hline 7 & 400 & $1,08 \pm 0,18$ & $0,65 \pm 0,05$ & 82,58 & 89,52 & 16,67 & 7,69 & Idem \\
\hline
\end{tabular}

\begin{tabular}{lr}
\hline Test de corrélation de Pearson (test paramétrique) : \\
\hline Valeur observée & 0,097 \\
p-value bilatérale & 0,855 \\
Alpha & 0,05 \\
\hline Conclusion : la corrélation n'est pas significative au seuil de 0,05
\end{tabular}


Tableau 2 : Résultats des essais de traitement avec des os calcinés de différentes granulométries.

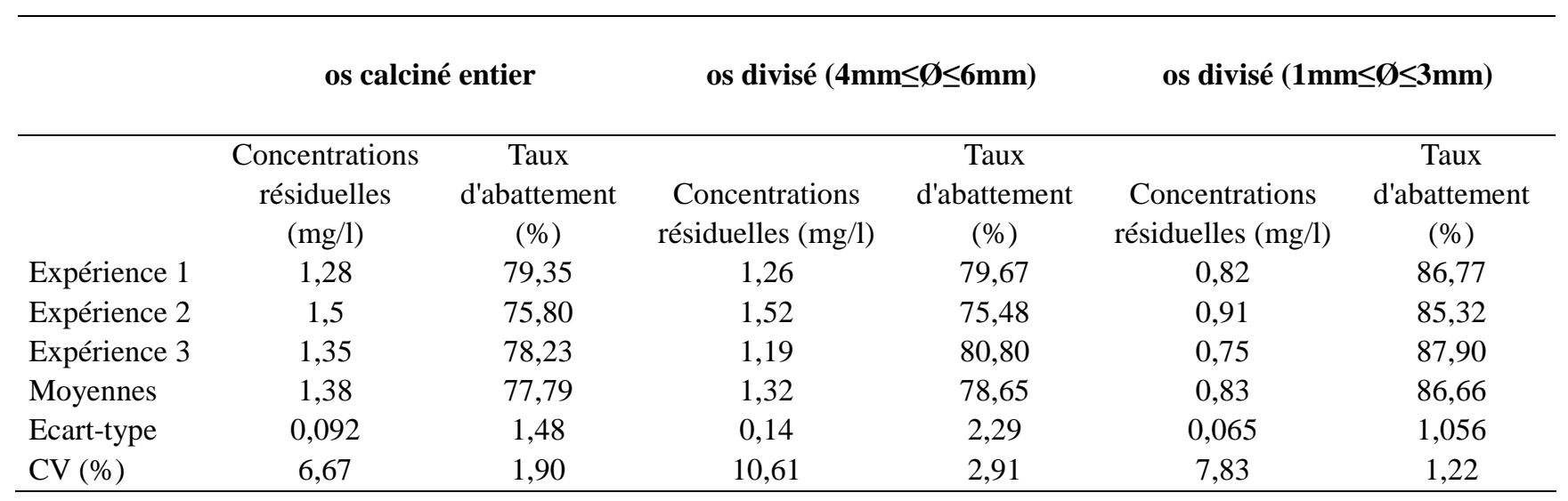




\section{DISCUSSION}

Les résultats des essais de traitement montrent que les eaux traitées avec les os calcinés à partir de $100{ }^{\circ} \mathrm{C}$ à $400{ }^{\circ} \mathrm{C}$ quel que soit le temps de calcination ne sont pas assez concluant. En effet, malgré que les concentrations finales en ions fluorures après 48 heures soient toutes conformes aux recommandations de l'OMS, nous observons une coloration jaune de l'eau traitée et la présence de matière lipidique. Par contre les eaux traitées avec les os calcinés à $500{ }^{\circ} \mathrm{C}$ et $625{ }^{\circ} \mathrm{C}$ donnent après 24 heures des concentrations en ions fluorures largement inférieurs à la valeur recommandée par l'OMS mais aussi ne présentent ni de coloration, ni de présence de matière lipidique.

Le suivi de la DCO de ces eaux traitées présentant une coloration, montre que la valeur de ce paramètre passait de $20 \mathrm{mg} / \mathrm{L}$ pour l'eau non traitée aux os calcinés à 745 $\mathrm{mg} / \mathrm{L}$ en moyenne pour les eaux traitées avec les os calcinés à des températures inférieures à $200{ }^{\circ} \mathrm{C}$. De cette observation, on peut penser que le chauffage de l'os a fondu et libéré la matière lipidique contenu dans la moelle osseuse. Ce qui apparaît en surface lors du traitement.

A partir de $200{ }^{\circ} \mathrm{C}$ à $300{ }^{\circ} \mathrm{C}$, on constate au niveau des eaux traitées que la coloration jaune s'est accentuée et la présence de matière lipidique a considérablement diminuée. Le suivi de la DCO montre que cette dernière est passée de $20 \mathrm{mg} / \mathrm{L}$ au niveau de l'échantillon d'eau non traité à $345 \mathrm{mg} / \mathrm{L}$ au niveau des eaux traitées. Cette baisse de la DCO des échantillons traités par rapport à l'expérience précédente est probablement due à une perte partielle et/ou une thermodégradation de la matière lipidique en matière organique hydrosoluble, lorsque la température est comprise entre 200 et $300{ }^{\circ} \mathrm{C}$. Ces produits de dégradation hydrosolubles de la matière grasse osseuse semblent influencer négativement l'abattement du taux des ions fluorures de l'eau.

$\mathrm{Au}$ delà de $400{ }^{\circ} \mathrm{C}$, l'eau redevient claire et la DCO repasse à la valeur initiale de $20 \mathrm{mg} / \mathrm{L}$. Une explication plausible à ce phénomène serait la disparition totale de la matière organique de l'os sous l'effet de l'augmentation de la température de calcination qui l'aurait dégradée entièrement redisposant plus l'os calcinés à la fixation des ions fluorures contenus dans l'eau à traiter suivant l'équation :

$\mathrm{Ca}^{2+}+2 \mathrm{~F}^{-} \rightleftarrows \mathrm{CaF}_{2}$

Cette situation a permis d'atteindre des teneurs minimales en ions fluorures en dessous de la limite recommandée par l'OMS. Diawara et al. (2004) ont rapporté des expériences de défluoruration concluante en Inde avec des os calcinés à $600{ }^{\circ} \mathrm{C}$ pendant 20 $\mathrm{mn}$. Mais malheureusement, dans notre cas, le choix des os calcinés à $625{ }^{\circ} \mathrm{C}$ pendant 24 heures a conduit à une augmentation sensible du $\mathrm{pH}$ de l'eau traitée qui est passé de 7,81 à 11,3 .

Cette variation du $\mathrm{pH}$ du milieu réactionnel constatée serait liée à la libération des ions $\mathrm{OH}^{-}$contenus dans l'hydroxyapathite des os utilisés. Ainsi, en dehors du phénomène d'adsorption couramment reconnu pour l'os calciné dans la défluoruration des eaux contaminées, un processus de défluoruration par substitution nucléophile des ions hydroxyle contenus dans l'hydroxyapathite par les ions fluorures de l'eau semble également se produire suivant l'équation :

\section{$\mathrm{Ca}_{5}\left(\mathrm{PO}_{4}\right)_{3}(\mathrm{OH})+\mathrm{F}^{-} \rightleftarrows \mathrm{Ca}_{5}\left(\mathrm{PO}_{4}\right)_{3}(\mathrm{~F})+\mathrm{OH}^{-}$}

En ce qui concerne le choix de la granulométrie, il ressort que les taux d'abattement évoluent en sens inverse à la granulométrie. Ceci peut s'expliquer par l'augmentation de la surface d'échange 
lorsqu'on divise plus ou moins finement l'os. Ainsi, le meilleur résultat a été obtenu avec des os calcinés divisés de diamètre compris entre 1 et $3 \mathrm{~mm}$. Mais, des études antérieures ont montré que l'utilisation d'os calciné pulvérisé (granulométrie très fine) altère les propriétés organoleptiques de l'eau traitée (augmentation de la turbidité et apparition d'une couleur blanchâtre difficilement éliminable) (Yoro et Nougbodohoué, 2007).

\section{Conclusion}

L'utilisation des os calcinés a permis de conduire avec succès des essais de défluoruration des eaux souterraines d'un forage de la commune de Dassa-zoumé. De nos essais il ressort que la température de calcination optimale des os pour un traitement efficace est de $500{ }^{\circ} \mathrm{C}$ pendant 24 heures. La granulométrie comprise entre 1 et $3 \mathrm{~mm}$ a donné les meilleurs résultats. Après un temps de défluoruration de 24 heures, le seuil recommandé par l'OMS est déjà atteint.

Toutefois, l'influence des paramètres comme la concentration initiale en ion fluorure, l'alcalinité, le pH de l'eau brute, les possibilités de régénération du matériel adsorbant et son coût doivent être étudiés pour améliorer l'efficacité et l'efficience de cette méthode de traitement.

\section{REFERENCES}

Ahokpossi Y. 2005. Qualité de l'eau dans la commune de Dassa-Zoumè : Cas des fluorures. Université d'Abomey-Calavi (Ecole Polytechnique d'Abomey-Calavi), République du Bénin, p. 72.

Amor Z, Bariou B, Mameri N, Taky M, Nicolas S, Elmidaoui A. 2001. Fluoride Removal from Brackich Water by Electrodialysis, Desalination. p. 215.

Boukari M. 1982. Contribution à l'étude hydrogéologique des régions du socle de l'Afrique intertropicale. L'hydrogéologie de la région de Dassa-Zoumè. Thèse de doctorat de l'Université Cheikh Anta Diop de Dakar, p. 131.

Chavassieux P, Meunier PJ. 1995. Bénéfices et risques des apports fluorés. Arch. Pediatr., 2(6): 568-572.

Degrémont. 1978. Memento Technique de l'Eau. Ed. Degrémont: Paris.

Diawara CK, Essi-Tome H, Lhassani A, Pontié M, Buisson H. 2004. Filtrations membranaires et qualité de l'eau de Boisson en Afrique : Cas du fluor au Sénégal. MSAS'2004, p 135.

Dovonon L. 2004. Suivi de la qualité des eaux souterraines dans les départements du Zou et des Collines. Rapport de mission SQE/DG-Eau, p. 22.

Koritnig S. 1963. Zur Geochemie Des Fluors Den Sedimenten. Fortchr. Geol. Rheinld. Westf., 10: 231.

Mazet P. 2002. Les eaux souterraines riches en fluorures dans le monde. DEA en science de l'eau dans l'environnement continental, Maison des sciences de Montpellier, Rapport de stage, p. 45.

OMS. 2002. "Fluoride in drinking water." Environmental health information section, WHO Headquarters Office in Geneva,www.who.int/environmental inf omation/lnfomation resourcesktmdocslFl uoride/fluoride

OMS. 2004. Guidelines for Drinking-Water Quality (3rd edition, Vol. 1). Recommendation : Geneva.

Pontié M, Schrotter J-C, Lhassani A, Diawara CK. 2006. Traitement des eaux destinées à la consommation humaine : élimination domestique et industrielle du fluor en excès. L'actualité chimique, $\mathrm{n}^{\circ}$ 301- 302.

Sorg TJ. 1978. Treatment technology to meet the interim primary drinking water regulation for inorganics. J.A.W.W.A., 40(2): $58-64$.

Travi Y. 1993. Hydrogéologie et hydrochimie des aquifères du Sénégal, hydrogéochimie du fluor dans les eaux 
souterraines, Mémoire $\mathrm{N}^{\circ} 95$, ISSN 0302 -2684 , p.161.

Wedepohl K H. 1974. Hand-book of Geochemistry (vol.II-4). SpringerVerlage: Berlin, Heidelberg, New York, P. 9K-1.

Xu-Guo-Xun. 1992. Fluoride removal from drinking water by activated aluminia with $\mathrm{CO}_{2}$ gas acidizing method. J. Water SRT. AQUA, 43( 2): 58 - 64 .

Yang CL, Dluhy R. 2002. Electro-chemical generation of aluminium sorbent for fluoride adsorption. Journal of Hazardous Materials, 94(3): 239.
Yoro FA, Noudogbèssi L. 2007. Evaluation de l'impact sanitaire dû à la consommation des eaux contaminées en fluorures dans la commune de DassaZoumè et essai de défluoration. Mémoire de fin cycle d'ingénieur de l'Ecole Polytechnique d'Abomey-Calavi, p. 74.

Zogo D, Bawa LM, Soclo HH, Atchèkpè D. 2010. Elimination de la couleur et de la turbidité d'une eau de surface par coagulation-floculation au sulfate d'aluminium : cas de la retenue d'eau de l'Okpara en République du Bénin. Int. J. Biol. Chem. Sci., 4(5): 1667-1675. 